\title{
Proposal for the teaching of the chemical control of supragingival biofilm ${ }^{\S}$
}

\author{
Rui Vicente Oppermann ${ }^{(a)}$ \\ Alex Nogueira Haas ${ }^{(b)}$ \\ German Eduardo Miguel Villoria ${ }^{(c)}$ \\ Laura Guimarães Primo(d) \\ Júnia M. Serra-Negra ${ }^{(\mathrm{e})}$ \\ Efigênia Ferreira e Ferreira(f) \\ Claudio Mendes Pannuti(g)
}

(a) Chair Professor; (b) Adjunct Professor - Department of Periodontics, Federal University of Rio Grande do Sul, Porto Alegre, RS, Brazil.

(c) Coordinator, Dentistry Course, Gama Filho University, Rio de Janeiro, RJ, Brazil.

(d) DDS, MS, PhD, Associate Professor, Department of Pediatric Dentistry and Orthodontics, Federal University of Rio de Janeiro, Rio de Janeiro, RJ, Brazil.

(e)DDS, MS, PhD, Associate Professor, Department of Pediatric Dentistry and Orthodontics, Federal University of Minas Gerais, Belo Horizonte, MG, Brazil.

(f) Associate Professor, Department of Preventive and Community Dentistry, School of Dentistry, Federal University of Minas Gerais, Belo Horizonte, MG, Brazil.

(g) Assistant Professor, Department of Periodontics, School of Dentistry, University of São Paulo, São Paulo, SP, Brazil.

\begin{abstract}
The mechanical control of supragingival biofilm is accepted as one of the most important measures to treat and prevent dental caries and periodontal diseases. Nevertheless, maintaining dental surfaces biofilm-free is not an easy task. In this regard, chemical agents, mainly in the form of mouthwashes, have been studied to help overcome the difficulties involved in the mechanical control of biofilm. The aim of this paper was to discuss proposals for the teaching of supragingival chemical control (SCC) in order to improve dentists' knowledge regarding this clinical issue. Firstly, the literature regarding the efficacy of antiseptics is presented, clearly showing that chemical agents are clinically effective in the reduction of biofilm and gingival inflammation when used as adjuvant agents to mechanical control. Thus, it is suggested that the content related to SCC be included in the curricular grid of dental schools. Secondly, some essential topics are recommended to be included in the teaching of SCC as follows: skills and competencies expected of a graduate dentist regarding SCC; how to include this content in the curricular grid; teaching-learning tools and techniques to be employed; and program content.
\end{abstract}

Descriptors: Anti-Infective Agents, Local; Education; Learning; Gingivitis; Biofilms.

\section{Introduction}

The objectives of dental practice include prevention and treatment of oral diseases that are most prevalent among populations. One of the main actions taken both for the prevention and the treatment of periodontal diseases and caries has been the mechanical control of supragingival biofilm. However, keeping dental surfaces biofilm-free is not an easy task. In order to aid in biofilm control, chemical agents have been studied over decades, especially in the form of mouthwashes. Sales of these chemical agents have been growing worldwide, and a significant number of commercial products with various active ingredients are currently available on the market. Particularly in Brazil, there is unrestricted access to mouthwashes at pharmacies and supermarkets, and this has been popularizing theses products and their continuous - and sometimes indiscriminate - use.

In this context, it is important for the dental professional to be acquainted with the agents and products designed for the chemical control of supraginigival biofilm, as well as to know how to indicate them, evalu-
Received for publication on Jul 05, 2010 Accepted for publication on Jul 15, 2010 
ate their effectiveness and any probable adverse effects. Dental professionals are expected to have these skills and competencies, which can only be achieved with the engagement of educational institutions in including these contents in their curricular grids in order to meet the great demand for information related to them. Thus, this work group has met in order to formulate proposals for the teaching of the chemical control of supragingival biofilm with the aim of improving dental professionals' education regarding this matter.

\section{Justification for the chemical control of supragingival biofilm and gingival inflammation}

Supragingival bacterial biofilm is considered one of the determining factors of the oral diseases that are most prevalent among populations - caries and periodontal disease. ${ }^{1,2}$ Therefore, treatment and prevention strategies should include the fight against this biofilm. Since biofilm represents an infectious component in the causal chain of oral diseases, it is plausible to apply chemical agents that act against the bacteria present in biofilm.

It is known that tooth brushing is a widely practiced habit. However, only a small percentage of people perform a high standard of mechanical control of supragingival biofilm. This is due either to the lack of motivation to perform hygiene procedures in all teeth or to issues related to manual skills. Difficulties in the mechanical control of dental biofilm are further aggravated when it comes to cleaning proximal surfaces. Dental floss or other interproximal cleansing methods are not widely used and require greater motivation and manual skill. This can also be confirmed by the limited quantity of dental floss consumed in several countries, including Brazil. ${ }^{3}$ In this respect, means of chemical control could be used together with mechanical control to improve the effectiveness of the daily removal of supragingival biofilm. ${ }^{4,5}$ Moreover, there are situations in which individuals are incapable to perform the mechanical control of supragingival biofilm. The most common situations include postoperative periods following oral surgery, traumas with or without intermaxillary immobilization, and physical and/or mental deficiencies.

It is in this context that scientific evidence has been produced on the chemical control of supragingival biofilm, and the main clinical outcomes are the reduction of the formation of biofilm and the reduction of gingival inflammation, assessed with the use of indexes accepted in the literature. Chlorhexidine has been studied since the last century ${ }^{6}$ and is recognized as the gold-standard chemical agent for biofilm control, ${ }^{7,8}$ especially due to its high substantivity (ability to remain active in the oral environment) and the antimicrobial activity against bacteria present in the oral cavity. Studies show reductions of up to $71 \%$ and $45 \%$ in biofilm and gingival inflammation, respectively, with the use of chlorhexidine in comparison with placebo. ${ }^{9}$ However, the use of chlorhexidine is limited due to the occurrence of important adverse effects, particularly tooth staining and transitory change in taste sensation. For this reason, chlorhexidine has been commonly indicated as a substitute for mechanical control only for short periods of time.

Various mouthwashes have been studied to help overcome the difficulties related to mechanical control and effectively reduce the formation of biofilm and gingivitis, with an emphasis on those containing triclosan, essential oils and cetylpyridinium chloride as active ingredients. Meta-analyses have consistently shown that the daily use of mouthwashes containing essential oils and cetylpyridinium chloride significantly reduces the visible plaque (biofilm) and gingivitis in comparison with the use of placebo solutions or with the non-use of any solution. ${ }^{10-12}$ Few studies have been conducted with solutions containing triclosan since this agent is found mostly in toothpaste formulations. Few studies have evaluated the use of cetylpyridinium chloride, showing statistically significant anti-plaque and anti-gingivitis effects, although of small clinical magnitude. ${ }^{11}$ Most of these studies were carried out evaluating the antiplaque and anti-gingivitis effect of essential oils. Literature indicates that there is a greater consistency in the studies showing a significant additional effect for essential oils when used together with unsupervised toothbrushing. ${ }^{10,12,13}$

Concerns have emerged regarding the risk of 
development of oral cancer due to the chronic and continuous use of mouthwashes containing alcohol in their formulation. ${ }^{14}$ This is due to the fact that the consumption of alcoholic beverages is a risk factor for the development of oral cancer, and people have indirectly questioned if the daily use of mouthwashes containing alcohol would have the same effect. However, there is no evidence that the use of the products available on the market could be associated to the development of oral cancer.

Few studies have been published about this matter, producing scientific evidence of low quality and yielding inconclusive ${ }^{15,16}$ findings, so it can be stated that mouthwashes containing alcohol are safe and effective. ${ }^{17}$

Literature clearly shows that chemical agents are clinically effective in the reduction of biofilm and gingival inflammation when used as supporting agents in mechanical control, so they must be indicated in clinical practice. In this respect, to fail to indicate the chemical control of biofilm means to fail to provide the patient with the best possible guidance. Consequently, the dentist must have adequate knowledge on the subject to be able to indicate the best chemical control alternative for each case. This is why we suggest that the content related to the chemical control of supragingival biofilm should be included in the curricular grid of dentistry courses in Brazil. We further suggest that some essential topics should be included in the teaching of chemical control, as shown below:

1. Skills and competencies expected of a graduate dental professional regarding the chemical control of supragingival biofilm

2. Including these contents in the curricular grid

3. teaching-learning tools and techniques to be employed

4. Course content

\section{Skills and competencies expected of a graduate dental professional}

a. Identifying Supragingival Chemical Control (SCC) needs in individuals or groups

b. Searching and critically evaluating literature related to individual or collective SCC needs

c. Selecting and prescribing the active ingredients most appropriate to the patient's needs

d. Knowing the risks and benefits of the agents selected

e. Being able to include the patient in the decisionmaking process regarding this selection

f. Following up and evaluating the results of the use of the agent selected

g. Analyzing the cost-benefit ratio

\section{Including these contents in the curricular grid}

a. Integrating SCC contents for the basic and applied areas

b. Including SCC contents in individual and collective activities

c. Including SCC in clinical routines and protocols to deal with different complexities

d. Practical activities related to clinical and microbiological evaluation of SCC

e. Research and extension activities related to SCC

\section{Teaching-learning tools and techniques to be employed}

a. Expositive lectures

b. Encouraging the use of active methodologies
I. Seminars
II. Clinical cases
III. Problem solving
IV.Using distance learning tools

\section{Course content}

a. Background

b. Justification of SCC

I. Reasons for using - mechanical control limitations, deftness and motivation, self-efficacy etc.

c. Contextual review of the knowledge about supragingival biofilm and gingival inflammation

d. Ideal properties of agents used for SCC

e. Agent types and classes

f. Mechanisms of action

g. Pharmacodynamics, toxicological and adverse effects

h. Laboratory and experimental evidence of the effect of chemical agents

i. Clinical evidence 
i. Clinical evidence of the use in patients with special needs

k. Clinical evidence of SCC in Periimplantar infections

I. Indications and contraindications

$\mathbf{m}$. Use of SCC in treated patients that are included

\section{References}

1. Takahashi N, Nyvad B. Caries ecology revisited: microbial dynamics and the caries process. Caries Res. 2008;42(6):40918.

2. Loe H, Theilade E, Jensen SB. Experimental gingivitis in man. J Periodontol. 1965 May-Jun;36:177-87.

3. Gjermo P, Rosing CK, Susin C, Oppermann R. Periodontal diseases in Central and South America. Periodontol 2000. 2002;29:70-8.

4. Barnett ML. The rationale for the daily use of an antimicrobial mouthrinse. J Am Dent Assoc. 2006 Nov;137 Suppl:16S21S.

5. Addy M. O uso de anti-sépticos na terapia periodontal. In: Lindhe J, Karring T, Lang NP, editores. Tratado de periodontia clínica e implantologia oral. $4^{\text {a }}$ ed. Rio de Janeiro: Guanabara Koogan; 2005. p. 450-77.

6. Gjermo P. Chlorhexidine in dental practice. J Clin Periodontol. 1974;1(3):143-52.

7. Addy M. Chlorhexidine compared with other locally delivered antimicrobials. A short review. J Clin Periodontol. 1986 Nov;13(10):957-64.

8. Jones CG. Chlorhexidine: is it still the gold standard? Periodontol 2000. 1997 Oct;15:55-62.

9. Paraskevas S. Randomized controlled clinical trials on agents used for chemical plaque control. Int J Dent Hyg. 2005 Nov;3(4):162-78.

10. Gunsolley JC. A meta-analysis of six-month studies of antiplaque and antigingivitis agents. J Am Dent Assoc. 2006 Dec;137(12):1649-57. in a preventive periodic maintenance program

n. Relevant and fundamental aspects of SCC prescription

I. Legal aspects (forms of presentation, dosage etc.)

o. Evaluation of the cost-benefit ratio

11. Haps S, Slot DE, Berchier CE, Van der Weijden GA. The effect of cetylpyridinium chloride-containing mouth rinses as adjuncts to toothbrushing on plaque and parameters of gingival inflammation: a systematic review. Int J Dent Hyg. 2008 Nov;6(4):290-303.

12. Stoeken JE, Paraskevas S, van der Weijden GA. The longterm effect of a mouthrinse containing essential oils on dental plaque and gingivitis: a systematic review. J Periodontol. 2007 Jul;78(7):1218-28.

13. Bauroth K, Charles CH, Mankodi SM, Simmons K, Zhao Q, Kumar LD. The efficacy of an essential oil antiseptic mouthrinse vs. dental floss in controlling interproximal gingivitis: a comparative study. J Am Dent Assoc. 2003 Mar;134(3):35965.

14. McCullough MJ, Farah CS. The role of alcohol in oral carcinogenesis with particular reference to alcohol-containing mouthwashes. Aust Dent J. 2008 Dec;53(4):302-5.

15. Carretero Pelaez MA, Esparza Gomez GC, Figuero Ruiz E, Cerero Lapiedra R. Alcohol-containing mouthwashes and oral cancer. Critical analysis of literature. Med Oral. 2004 Mar-Apr;9(2):120-3, 116-20.

16. Cole P, Rodu B, Mathisen A. Alcohol-containing mouthwash and oropharyngeal cancer: a review of the epidemiology. J Am Dent Assoc. 2003 Aug;134(8):1079-87.

17. Silverman S Jr, Wilder R. Antimicrobial mouthrinse as part of a comprehensive oral care regimen. Safety and compliance factors. J Am Dent Assoc. 2006 Nov;137 Suppl:22S-26S. 